\title{
METRIC PROPERTIES OF THE CYLINDER OF KUBOTA
}

\author{
RUTH B. RASMUSEN
}

1. Introduction. It is the purpose of this note to derive some properties of the surface normal and the superosculating lines on an analytic surface $S$ by means of a parabolic cylinder which Kubota has defined* $^{*}$ and has used for studying the properties of the affine normal to a surface and the curves of Darboux and Segre in affine differential geometry. If we consider all of the sections of an analytic surface $S$ made by planes passing through the same tangent $t$, the locus of the parabolas which osculate these sections at the common point of contact $P$ is a parabolic cylinder which we shall call the cylinder of Kubota.

In what follows we shall adhere quite closely to the notation used in Chapter 6 of Lane's Projective Differential Geometry of Curves and Surfaces, Chicago, 1932.

2. Analytic basis. It is convenient to take the lines of curvature for the parametric curves and to employ a local trihedron at a point of the surface whose edges are the tangents of the lines of curvature and the normal of the surface at the point. This section is designed to introduce these concepts and to collect some formulas which will be used later on in this note.

Let us consider in ordinary metric space a non-developable surface, not a sphere, whose parametric equations in cartesian coordinates are

$$
x=x(u, v), \quad y=y(u, v), \quad z=z(u, v) .
$$

Let the lines of curvature be the parametric curves on this surface. Then its first and second fundamental forms, written in the customary notation, are

$$
E d u^{2}+G d v^{2}, \quad D d u^{2}+D^{\prime \prime} d v^{2} .
$$

The principal radii of normal curvature $R_{1}, R_{2}$ at a point of the surface are defined by the formulas $R_{1}=E / D, R_{2}=G / D^{\prime \prime}$.

As a local trihedron of reference at a point $(x, y, z)$ of the surface $S$,

* T. Kubota, Einige Bemerkungen zur Affinflächentheorie, The Science Reports of the Tôhoku Imperial University, (1), vol. 19 (1930), p. 163. See also T. Kubota, Einige Bemerkungen zur Affinflächentheorie, Japanese Journal of Mathematics, vol. 10 (1933), p. 217. 
we shall take the origin at this point, the $\xi$-axis along the $u$-tangent, the $\eta$-axis along the $v$-tangent, and the $\zeta$-axis along the surface normal.

The power series expansions for the local coordinates $\xi, \eta, \zeta$ of a point near a point $P(0,0,0)$ on a surface $S$ in terms of the increments $\Delta u, \Delta v$ corresponding to displacement on $S$ from the point $(0,0,0)$ to the point $(\xi, \eta, \zeta)$ are

$$
\begin{aligned}
\xi= & E^{1 / 2}\left[\Delta u+\frac{1}{2}\left(\{11,1\} \Delta u^{2}+2\{12,1\} \Delta u \Delta v+\{22,1\} \Delta v^{2}\right)+\cdots\right], \\
\eta= & G^{1 / 2}\left[\Delta v+\frac{1}{2}\left(\{11,2\} \Delta u^{2}+2\{12,2\} \Delta u \Delta v+\{22,2\} \Delta v^{2}\right)+\cdots\right], \\
\zeta= & \frac{1}{2}\left(D \Delta u^{2}+D^{\prime \prime} \Delta v^{2}\right)+\frac{1}{6}\left[\left(D_{u}+\{11,1\} D\right) \Delta u^{3}+3\{12,1\} D \Delta u^{2} \Delta v\right. \\
& \left.+3\{12,2\} D^{\prime \prime} \Delta u \Delta v^{2}+\left(D_{v}^{\prime \prime}+\{22,2\} D^{\prime \prime}\right) \Delta v^{3}\right]+\cdots .
\end{aligned}
$$

From the expansions (1) it is possible to compute an expansion for $\zeta$ as a power series in $\xi, \eta$ with undetermined coefficients and then demand that the expansions for $\xi, \eta, \zeta$ in (1) shall satisfy this equation identically in $\Delta u, \Delta v$ as far as the terms of any desired order. Thus we find, to terms of the third order,

(2) $\zeta=\frac{1}{2}\left(\frac{\xi^{2}}{R_{1}}+\frac{\eta^{2}}{R_{2}}\right)+\frac{1}{6}\left(\alpha_{0} \xi^{3}+3 \alpha_{1} \xi^{2} \eta+3 \alpha_{2} \xi \eta^{2}+\alpha_{3} \eta^{3}\right)+\cdots$,

where

$$
\begin{aligned}
\alpha_{0}=\frac{1}{E^{1 / 2}}\left(\frac{1}{R_{1}}\right)_{u}, \quad \alpha_{1} & =\frac{1}{G^{1 / 2}}\left(\frac{1}{R_{1}}\right)_{v}, \quad \alpha_{2}=\frac{1}{E^{1 / 2}}\left(\frac{1}{R_{2}}\right)_{u}, \\
\alpha_{3} & =\frac{1}{G^{1 / 2}}\left(\frac{1}{R_{2}}\right)_{v} .
\end{aligned}
$$

3. The equation of the cylinder of Kubota. In order to find the equation of the parabolic cylinder of Kubota, let us regard a curve $C$ as imbedded in the one-parameter family of curves on the surface $S$ defined by the equation $d v-\lambda d u=0$, where $\lambda$ is a function of $u, v$. The tangent $t$ of the curve $C$ at point $P$ has the equations $\zeta=\eta-A \xi$ $=0$, where

$$
A=\lambda\left(\frac{G}{E}\right)^{1 / 2} .
$$

If we cut the surface (2) by the plane

$$
\zeta=n(\eta-A \xi),
$$

not passing through an asymptotic tangent, then the equation of the parallel projection of the curve of section in the direction of the $\zeta$-axis on the tangent plane is 


$$
n(\eta-A \xi)=\frac{1}{2}\left(\frac{\xi^{2}}{R_{1}}+\frac{\eta^{2}}{R_{2}}\right)+\frac{1}{6} \phi_{3}(\xi, \eta)+\cdots .
$$

It is convenient to make the following definition of $\phi_{3}(\xi, \eta)$ :

$$
\phi_{3}(\xi, \eta)=\alpha_{0} \xi^{3}+3 \alpha_{1} \xi^{2} \eta+3 \alpha_{2} \xi \eta^{2}+\alpha_{3} \eta^{3} .
$$

If $n \neq 0$, equation (4) is representable as a power series in the form

$$
\eta=A \xi+B \xi^{2}+C \xi^{3}+\cdots
$$

in which the coefficients have the following values:

$$
A=\lambda\left(\frac{G}{E}\right)^{1 / 2}, \quad n B=\frac{1}{2}\left(\frac{1}{R_{1}}+\frac{A^{2}}{R_{2}}\right), \quad n C=\frac{A B}{R_{2}}+\frac{1}{6} \phi_{3}(1, A) .
$$

The equation of the osculating parabola at $P$ of the curve (5) is

$$
p \xi^{2}+q \xi \eta+r \eta^{2}+\eta-A \xi=0
$$

in which the coefficients are given by $p=A^{2} r+\left(A C-B^{2}\right) / B, \quad q=-(2 A B r+C) / B, \quad r=-C^{2} / 4 B^{3}$.

If we eliminate $n$ between equations (3) and (6), we obtain

$$
\begin{aligned}
{\left[3\left(\xi / R_{1}+A \eta / R_{2}\right)\left(1 / R_{1}+A^{2} / R_{2}\right)+\right.} & \left.\phi_{3}(1, A) \zeta\right]^{2} \\
& -18 \zeta\left(1 / R_{1}+A^{2} / R_{2}\right)^{3}=0,
\end{aligned}
$$

which is the equation of the parabolic cylinder of Kubota.

4. Properties. The equation of the diametral plane of cylinder (7) which passes through the point $P$ is

$$
3\left(\xi / R_{1}+A \eta / R_{2}\right)\left(1 / R_{1}+A^{2} / R_{2}\right)+\phi_{3}(1, A) \xi=0 .
$$

We now proceed to prove the following theorem:

If the tangent $t$ is permitted to vary about the point $P$, the diametral plane passing through the point $P$ of this parabolic cylinder envelops an algebraic cone of the fourth order and the third class with three cusp edges which intersect in the line of centers of the quadrics of Darboux. Three tangent planes intersect in the surface normal. The three directions of the tangent $t$ to which these tangent planes correspond are the tangents of the superosculating lines, and these tangent planes give as the lines of intersection with the tangent plane the harmonic conjugates of the tangents of the superosculating lines.

In order to obtain the equation of the enveloping cone of plane (8) in tangential coordinates, we set 


$$
\begin{aligned}
& \rho u_{1}=\left(1 / R_{1}+A^{2} / R_{2}\right) / R_{1}, \\
& \rho u_{2}=A\left(1 / R_{1}+A^{2} / R_{2}\right) / R_{2}, \\
& \rho u_{3}=\phi_{3}(1, A) / 3 .
\end{aligned}
$$

Eliminating $\rho$ and $\lambda$ homogeneously from the above equations, we obtain

$$
3 G^{1 / 2} R_{1}\left(G R_{1} u_{1}^{2}+E R_{2} u_{2}^{2}\right) u_{3}=\phi_{3}\left(G^{1 / 2} R_{1} u_{1}, E^{1 / 2} R_{2} u_{2}\right) .
$$

Consequently the cone is of class three.

The equation of the enveloping cone of plane (8) in point coordinates is obtained by eliminating $\lambda$ between equation (8) and the equation obtained by differentiating equation (8) with respect to $\lambda$. The equation of the cone in point coordinates is

$$
\left(a \frac{E}{G}\right)^{3}-\left[4 b^{3} d-27 a^{2} d^{2}+b^{2} c^{2}-4 a c^{3}+18 a b c d\right]=0,
$$

in which $a, b, c, d$ are defined by

$$
\begin{aligned}
a=\frac{\eta}{R_{2}{ }^{2}}+\frac{\alpha_{3} \zeta}{3}, \quad b & =\frac{\xi}{R_{1} R_{2}}+\alpha_{2} \zeta, \quad c=\frac{\eta}{R_{1} R_{2}}+\alpha_{1} \zeta, \\
d & =\frac{\xi}{R_{1}^{2}}+\frac{\alpha_{0} \zeta}{3} .
\end{aligned}
$$

Neglecting the factor $a$, we see that the cone is of the fourth degree in $\xi, \eta, \zeta$. The planes $a=0$ and $d=0$ are tangent planes, and the lines $a=b=0$ and $c=d=0$ are generators, of cone (9).

When a surface is referred to its lines of curvature, the curves which possess the property that the normal sections in the directions of these curves at a point are straight lines, or are superosculated by their circles of curvature, are called the superosculating lines* of the surface. By direct computation it can be shown that as $n \rightarrow \infty$ the directions for which curve (4) is superosculated by its osculating circle are given by

$$
\phi_{3}(1, A)=0 .
$$

Using the definition of the superosculating lines, and verifying that the harmonic conjugate of $\zeta=\eta-A \xi=0$ is $\zeta=\xi / R_{1}+A \eta / R_{2}=0$, we obtain the above theorem.

Wisconsin State Teachers College

* Eisenhart, Differential Geometry, Boston, 1909, p. 187. 